\title{
Intramolecular vibrational energy redistribution in bridged azulene- anthracene compounds: Ballistic energy transport through molecular chains
}

\author{
D. Schwarzer, P. Kutne, C. Schröder, and J. Troe \\ Max-Planck-Institut für Biophysikalische Chemie, Am Fassberg, 37077 Göttingen, Germany
}

(Received 17 February 2004; accepted 3 May 2004)

\begin{abstract}
Intramolecular vibrational energy flow in excited bridged azulene-anthracene compounds is investigated by time-resolved pump-probe laser spectroscopy. The bridges consist of molecular chains and are of the type $\left(\mathrm{CH}_{2}\right)_{m}$ with $m$ up to 6 as well as $\left(\mathrm{CH}_{2} \mathrm{OCH}_{2}\right)_{n}(n=1,2)$ and $\mathrm{CH}_{2} \mathrm{SCH}_{2}$. After light absorption into the azulene $S_{1}$ band and subsequent fast internal conversion, excited molecules are formed where the vibrational energy is localized at the azulene side. The vibrational energy transfer through the molecular bridge to the anthracene side and, finally, to the surrounding medium is followed by probing the red edge of the azulene $S_{3}$ absorption band at $300 \mathrm{~nm}$ and/or the anthracene $S_{1}$ absorption band at $400 \mathrm{~nm}$. In order to separate the time scales for intramolecular and intermolecular energy transfer, most of the experiments were performed in supercritical xenon where vibrational energy transfer to the bath is comparably slow. The intramolecular equilibration proceeds in two steps. About $15 \%-20 \%$ of the excitation energy leaves the azulene side within a short period of 300 fs. This component accompanies the intramolecular vibrational energy redistribution (IVR) within the azulene chromophore and it is caused by dephasing of normal modes contributing to the initial local excitation of the azulene side and extending over large parts of the molecule. Later, IVR in the whole molecule takes place transferring vibrational energy from the azulene through the bridge to the anthracene side and thereby leading to microcanonical equilibrium. The corresponding time constants $\tau_{\text {IVR }}$ for short bridges increase with the chain length. For longer bridges consisting of more than three elements, however, $\tau_{\mathrm{IVR}}$ is constant at around 4-5 ps. Comparison with molecular dynamics simulations suggests that the coupling of these chains to the two chromophores limits the rate of intramolecular vibrational energy transfer. Inside the bridges the energy transport is essentially ballistic and, therefore, $\tau_{\mathrm{IVR}}$ is independent on the length. (C) 2004 American Institute of Physics. [DOI: 10.1063/1.1765092]
\end{abstract}

\section{INTRODUCTION}

Intramolecular vibrational energy redistribution (IVR) is an important step of reactive processes in chemistry. ${ }^{1}$ The assumption of rapid IVR inside a molecule prior to reaction, on the one hand, is essential for the applicability of statistical rate theories. ${ }^{2,3}$ If randomization of vibrational energy is hindered by internal bottlenecks such that reaction is faster than IVR, on the other hand, the system behaves nonergodically which allows for mode specific chemistry. Hence, great efforts have been expended over the last decade to elucidate the mechanisms and principles determining IVR.

Most information on IVR was derived from frequencyresolved experiments on isolated molecules in low pressure gases or supersonic jets (see Refs. 1, 4, and 5, and references cited therein). Details of IVR mechanisms here are deduced by resolving features and analyzing widths, splittings, and relative intensities of lines within spectral clumps associated with zero-order bright states. According to these results and theoretical studies, IVR occurs stepwise among subsets of modes (polyades) within the vibrational manifold. Frequency-domain experiments on IVR are limited to the gas phase mostly at low temperatures. In liquids two-color pump-probe techniques with high time resolution have advantages and offer the opportunity to directly follow the population decay of zero-order bright states or to indepen- dently interrogate vibrational states populated in the course of a relaxation sequence. ${ }^{6-10}$ In the presence of a bath, vibrational energy not only is transferred finally to the surrounding but also the IVR process itself can be accelerated by anharmonic couplings introduced into the reactant by collisions. ${ }^{11,12}$

In the present paper we address the question how fast vibrational energy is transferred through molecular chains which at each side are connected to relatively large chromophores which also act as heat reservoirs. The employed molecules are bridged azulene-anthracene compounds such as shown in Fig. 1. In the experiment one of these chromophores (the azulene part) is vibrationally excited by a short laser pulse; subsequently the time for reaching intramolecular equilibrium, by transfer of energy through the molecular chain to the other chromophore (the anthracene part), is measured. This study of IVR across molecular chains is related to the classical work of Rabinovitch and colleagues ${ }^{13-15}$ who prepared instable molecules or chemically activated radicals which were excited at a specific site. IVR rates in their work were estimated from relative rate measurements, calibrating pressure dependent product yields against the frequency of deactivating collisions. More recently, vibrational energy transfer across aliphatic alcohol molecules of different length has also been reported using 


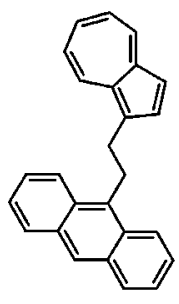

I

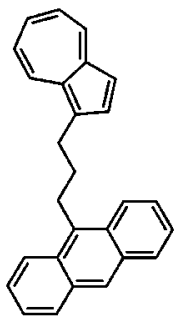

II

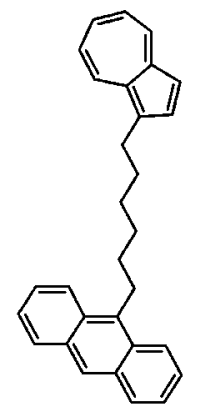

III

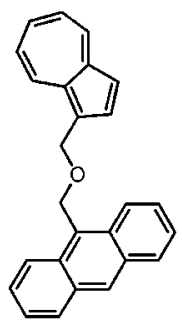

IV

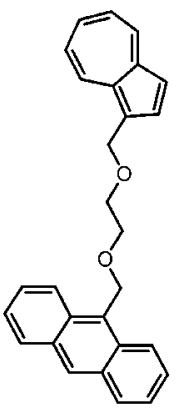

$\mathbf{V}$

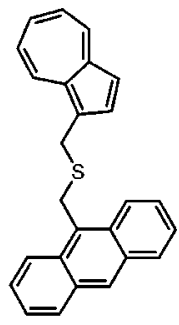

VI

FIG. 1. Molecular structures of the investigated compounds (I, 9-(2-azulene-1-yl-ethyl)-anthracene; II, 9-(3-azulene-1-yl-propyl)-anthracene; III, 9-(6azulene-1-yl-hexyl)-anthracene; IV, 9-(azulene-1-yl-methoxymethyl)-anthracene; V, 9-[2-(azulene-1-yl-methoxy)-ethoxymethyl]-anthracene; VI, 9-(azulene1-yl-methyl-sulfanylmethyl)-anthracene).

time-resolved spectroscopy. ${ }^{16}$ In this study the $\mathrm{OH}$ stretching vibration was excited by midinfrared laser pulses and the energy flow to the terminal $\mathrm{CH}_{3}$ group was followed by spontaneous anti-Stokes Raman scattering. Our measurements of vibrational energy transfer through molecular chains, in principle, resembles a classical macroscopic heat conduction experiment in which two heat reservoirs of different temperatures are connected by a heat-conducting rod. The heat-conducting links of the compounds shown in Fig. 1, however, unlike macroscopic materials are one-dimensional chains. The question, under which conditions Fourier's law of heat conduction applies also on a molecular scale, is of considerable theoretical interest. ${ }^{17,18}$ The influence of anharmonicity, disorder, system size, and presence of external potentials on the energy transport properties of onedimensional chains, in particular, has been investigated by molecular dynamics ${ }^{19-23}$ and quantum mechanical calculations. ${ }^{24,25}$

\section{EXPERIMENTAL SECTION}

\section{A. Experimental approach}

Selective vibrational excitation of the azulene part of compounds I-VI (Fig. 1) is achieved by absorption of a short laser pulse into the azulene $S_{1} \leftarrow S_{0}$ transition at $580 \mathrm{~nm}$. This initial electronic excitation is followed by fast internal conversion leaving the molecules in their ground electronic states with an excess vibrational energy of $17240 \mathrm{~cm}^{-1}$ localized at the azulene chromophore. For bare azulene, internal conversion is known to proceed within 1 ps or less; ${ }^{26-28}$ for substituted azulenes of the type discussed here, internal conversion times have also been reported to be very short such that vibrational excitation here is complete in less than $1 \mathrm{ps} .{ }^{29}$ The subsequent spreading of excess vibrational energy through the bridge over the whole molecule was followed by monitoring the energy loss of the azulene chromophore using the hot band absorption technique. In some cases the energy gain at the anthracene side was also monitored. Our method takes advantage of the fact that electronic absorption spectra broaden when the vibrational energy of the molecule is increased and Franck-Condon-active modes coupling to the electronic transition are excited. Often the broadening only depends on the average vibrational energy of the excited polyatomic molecules. ${ }^{30}$ Therefore, thermal spectra can also be used to calibrate the energy content of molecules which are far from equilibrium. For azulene and anthracene, these spectra are available in the range of the $S_{3} \leftarrow S_{0}$ and $S_{1} \leftarrow S_{0}$ transitions, ${ }^{29}$ respectively. In Fig. 2(a) for instance, the temperature dependence of the azulene gasphase absorption spectrum is shown. At the same time, spectra of thermally and optically heated molecules are shown to coincide when the average vibrational energies are identical. From these spectra the energy dependence of the azulene absorption coefficient $\epsilon_{\lambda}$ at selected wavelength $\lambda$ can be
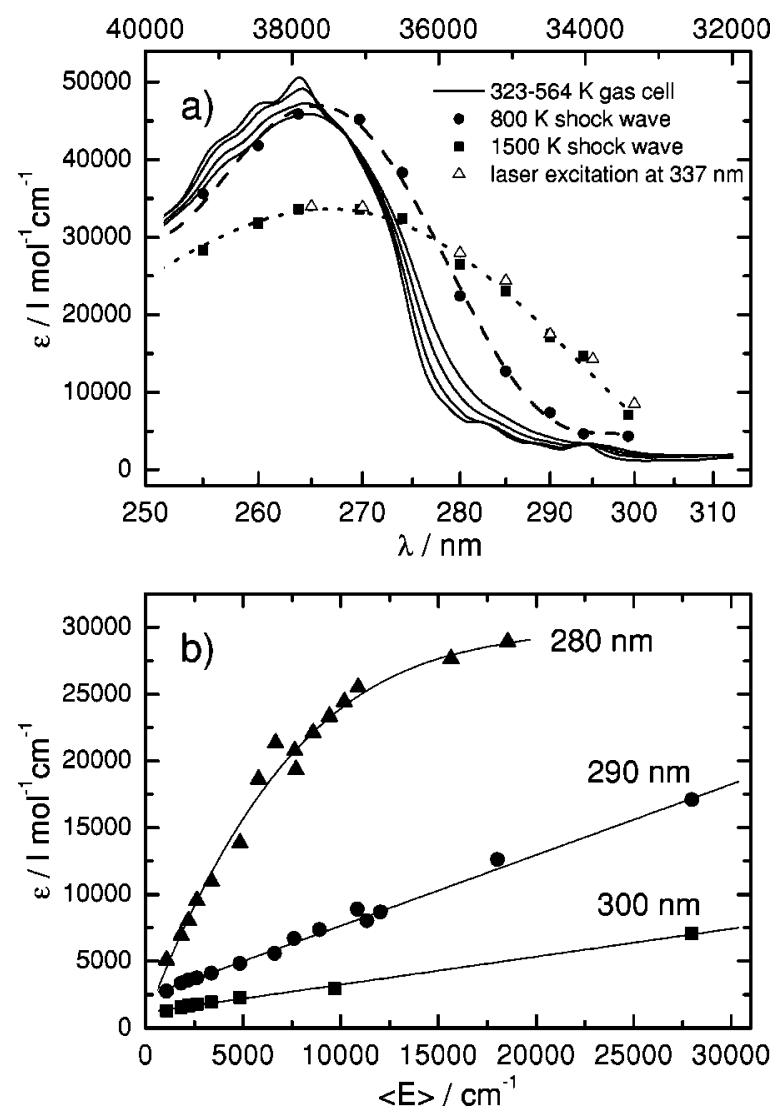

FIG. 2. (a) Gas-phase absorption spectra of azulene at various temperatures (laser excitation at $337 \mathrm{~nm}$ corresponds to $1580 \mathrm{~K}$ ); (b) energy dependence of the azulene absorption coefficient at selected wavelengths (from Refs. 31 and 47). 


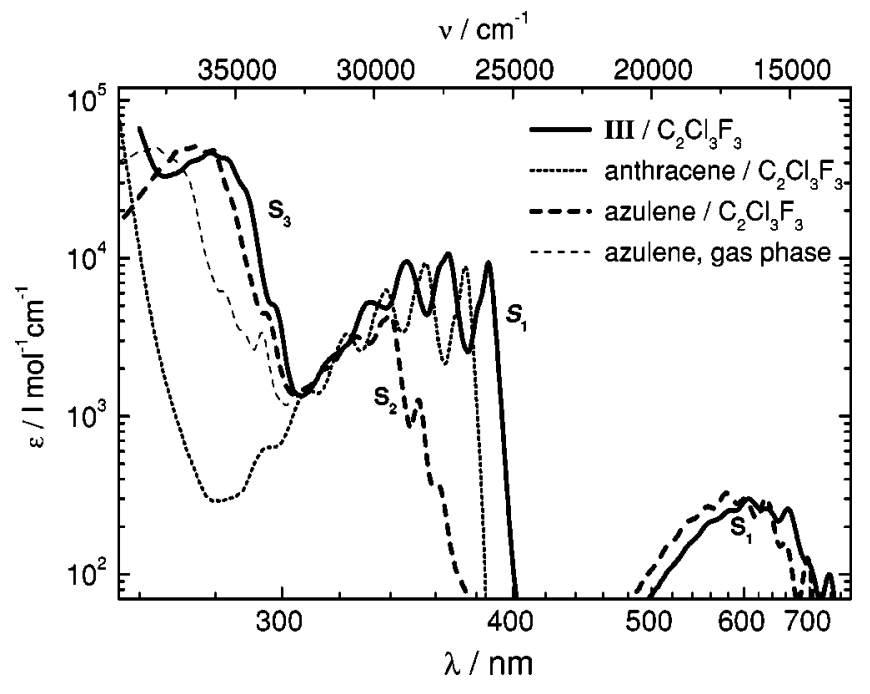

FIG. 3. Absorption spectrum of III in $\mathrm{C}_{2} \mathrm{Cl}_{3} \mathrm{~F}_{3}$ (spectra of azulene and anthracene in $\mathrm{C}_{2} \mathrm{Cl}_{3} \mathrm{~F}_{3}$, as well as azulene in the gas phase are shown for comparison; azulene and anthracene transitions are indicated by bold and italic letters, respectively).

derived [see Fig. 2(b)]. Note that at $290 \mathrm{~nm}$ the energy dependence $\epsilon_{290}(\langle E\rangle)$ is linear, i.e., transient absorption signals obtained at this wavelength directly reflect the azulene vibrational energy as a function of time. When measuring the azulene energy in our experiments, the probe wavelength was always chosen to be in this linear $\epsilon(\langle E\rangle)$ regime. Analogous spectral information is also available for the anthracene $S_{1} \leftarrow S_{0}$ absorption band. ${ }^{29}$

Probing the energy of the two chromophores separately also requires that the considered absorption bands of azulene and anthracene are well separated. This is actually the case here such as shown in Fig. 3 where a spectrum of III is compared with the spectrum of bare azulene and anthracene. The electronic absorption spectra of the other compounds, apart from small spectral shifts, are all very similar. In our experiments we probed the red edge of the $S_{3} \leftarrow S_{0}$ transition at about $300 \mathrm{~nm}$ to monitor the energy of the azulene chromophore. This absorption is just in the gap between the $S_{1}$ and $S_{2}$ bands of anthracene. Similarly, the gain of vibrational energy at the anthracene side, which arises from IVR through the bridge, can be measured at the red wing of the anthracene $S_{1} \leftarrow S_{0}$ absorption at about $400 \mathrm{~nm}$. This band reaches more than $20 \mathrm{~nm}$ into the gap between the $S_{1}$ and $S_{2}$ bands of azulene. When azulene and anthracene are combined in a single molecule like III, the considered transitions appear well separated and can be used to monitor either the energy loss of azulene at its $S_{3}$ band or the energy gain of anthracene at its $S_{1}$ band. Apart from small spectral shifts, the spectrum of III in Fig. 3 can be considered as the sum of the individual azulene and anthracene spectra with well separated bands. The spectral shifts of the considered electronic absorption bands are caused by solvatochromism in solution and by chemical substitution, i.e., by replacement of one hydrogen atom of the chromophores by a carbon chain. For our later analysis, we assume that the hot azulene spectra of Fig. 2(a) undergo the same spectral shifts as the cold spectra. This assumption has been made before ${ }^{29,31-33}$ and always led to an internally consistent analysis. Following the described technique, the gas-phase calibration curves of Fig. 2(b) can be used to transform absorption signals into energy-time profiles. The azulene $S_{3}$ band of compound III in Fig. 3, e.g., is redshifted by $16 \mathrm{~nm}$ with respect to bare azulene in the gas phase. This means that a probe wavelength of $\lambda_{\mathrm{pr}}=(290$ $+16) \mathrm{nm}=306 \mathrm{~nm}$ has to be used in order to measure the azulene energy of III in the linear $\epsilon(\langle E\rangle)$ regime such as represented by $\epsilon_{290}(\langle E\rangle)$ in Fig. 2(b).

All the experiments presented here were performed in solution. Thus, after intramolecular equilibration, the vibrational energy is finally transferred to the surrounding bath. To separate intramolecular and intermolecular energy transfer it is useful to choose a solvent for which vibrational energy transfer is particularly slow. In our previous study, we found that 1,1,2-trichloro-trifluoro-ethane $\left(\mathrm{C}_{2} \mathrm{Cl}_{3} \mathrm{~F}_{3}\right)$ is appropriate. ${ }^{29}$ In the present work, in addition we used supercritical xenon which combines energy transfer inefficiency with excellent solvent capability.

\section{B. Experimental technique}

The required laser pulses were generated employing a home-built Ti:sapphire oscillator-regenerative amplifier (RGA) system which produced 80 fs laser pulses at a repetition rate of $1 \mathrm{kHz}$ with energies of about $0.6 \mathrm{~mJ}$ centered at $800 \mathrm{~nm}$. A fraction of the pulse energy was frequency doubled and used to operate a one-stage nonlinearly pumped optical parametric amplifier. ${ }^{34}$ The resulting laser pulses at 550-620 nm were used to excite the azulene chromophore into the $S_{1}$ state. Part of the energy was frequency doubled to probe the internal energy of the azulene part at the red edge of the $S_{3} \leftarrow S_{0}$ transition at $290-310 \mathrm{~nm}$. Pulses at $\sim 400 \mathrm{~nm}$ required to probe the internal energy of the anthracene chromophore were generated by frequency doubling of the remaining light from the RGA. Typical energies of pump and probe pulses were about 3 and less than $0.05 \mu \mathrm{J}$, respectively. A computer-controlled translation stage adjusted the time delay between pump and probe pulses. The relative plane of polarization of both laser pulses was $54.7^{\circ}$ to avoid signal contributions arising from rotational relaxation. Lenses with focal lengths of $100 \mathrm{~mm}$ were used to focus the laser beams collinearly into the sample cell. Probe energies were measured in front of and behind the sample by means of photodiodes. Behind the sample an interference filter blocked transmitted excitation light. Every second pump pulse was blocked by a synchronized chopper to independently measure eventual drifts of the background absorption. The width of the cross correlation between pump and probe pulses was $150-200 \mathrm{fs}$. This is well below the time resolution of the experiment which is essentially limited by the $S_{1}$ internal conversion times of the azulene chromophore (0.6$1.0 \mathrm{ps})$.

Measurements in liquid solution at ambient conditions were performed in a $2 \mathrm{~mm}$ quartz flow cell. Experiments in supercritical xenon were carried out in a stainless steel high pressure cell with sapphire windows of $2 \mathrm{~mm}$ thickness. The optical path length inside the cell also was $2 \mathrm{~mm}$. Within the cell the sample solution was circulated using a small magnetic stirrer. ${ }^{35,36}$ Experiments were performed at room tem- 


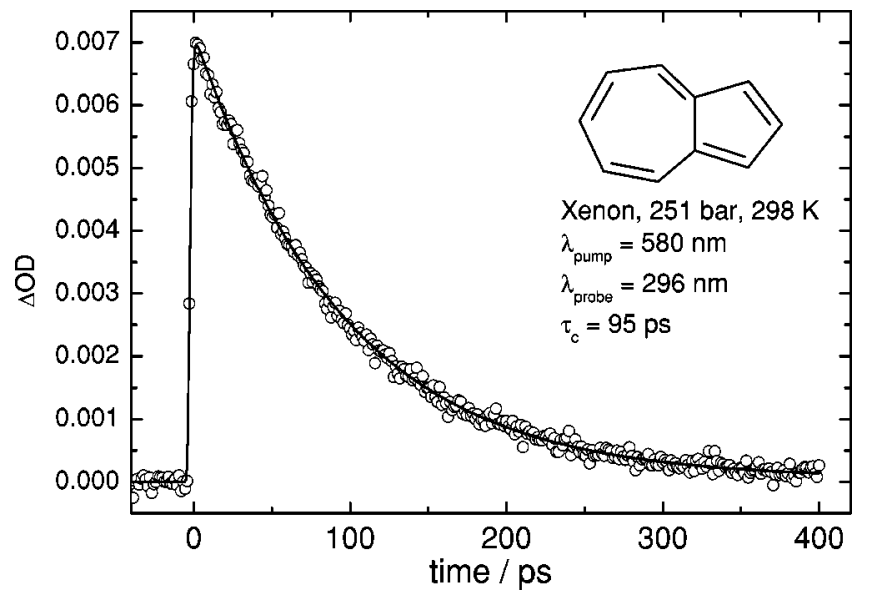

FIG. 4. Absorption-time profile recorded during the collisional energy transfer of azulene in xenon at 251 bar and $298 \mathrm{~K}$. The full line represents a fit assuming an exponential energy decay.

perature and pressures of 200-1000 bar which were generated with a high performance liquid chromatography pump connected to the high pressure cell. Over this range xenon behaves like a liquid and its density change is only $30 \%$. In all experiments the concentration of the azulene-anthracene compounds was 1-2 $\mathrm{mM}$.

All bridged azulene-anthracene compounds were synthesized in our laboratory. Compounds I-III were prepared by reaction of $\omega$-anthracene-9-yl-alkanal with 3 -methoxycarbonyl- $2 H$-cyclohepta[ $b]$ furan-2-one employing the enamine method followed by demethoxycarbonylation with $100 \%$ phosphoric acid. ${ }^{37}$ The $\omega$-anthracene-9-ylalkanals were synthesized by reaction of anthrone with $\omega$-chloroalkanols ${ }^{38}$ and subsequent oxidation of the formed $\omega$-anthracene-9-yl-alkanols with Dess-Martin periodinane. ${ }^{39}$ Starting material for the preparation of (thio)ethers IV-VI was 1-azulylmethyltrimethylammonium iodide which was treated with the sodium salts of the respective anthracene-9-yl-(thio)alcohols. ${ }^{40}$ After column chromatography the final purity of compounds I-VI was $>95 \%$ as controlled by ${ }^{1} \mathrm{H}$ and ${ }^{13} \mathrm{C}$ NMR spectroscopy. The solvents 1,1,2-trichloro-trifluoro-ethane (Aldrich, >99.9\%) and xenon (Messer-Griesheim, >99.99\%) could be used without purification.

\section{RESULTS}

\section{A. Time-resolved absorption signals}

In this section we illustrate the experimental observations of intramolecular and intermolecular vibrational energy transfer of I-VI. A more basic analysis follows in the following section. Before presenting results for the bridged compounds, in Fig. 4 we show a signal illustrating the energy loss by intermolecular vibrational energy transfer of the bare azulene molecule in supercritical xenon. Since collisional energy transfer (CET) is also observed for the bridged compounds, this signal serves as a reference. In xenon at $298 \mathrm{~K}$ and 251 bar, the solvatochromic shift of the azulene $S_{3}$-absorption band is about $6 \mathrm{~nm}$. Since a probe wavelength of $\lambda_{\mathrm{pr}}=296 \mathrm{~nm}$ was used, the shown signal is proportional to

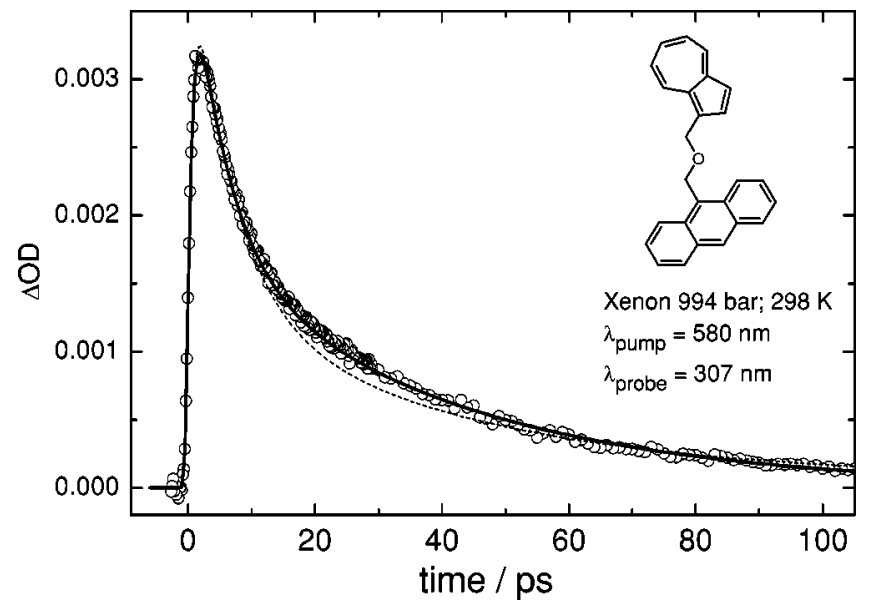

FIG. 5. Absorption-time profile of IV in xenon at 994 bar and $298 \mathrm{~K}$ probing the vibrational energy content in the azulene chromophore [full line, fit assuming a biexponential energy decay of Eq. (3) with amplitude ratio $A_{r}=E_{\mathrm{IVR}} / E_{\mathrm{CET}}$; dashed line, with $A_{r}=E_{\mathrm{intra}} / E_{\mathrm{CET}}$ (see text and Table I) $]$.

the energy content of the azulene molecule. After $S_{1}$ excitation at $580 \mathrm{~nm}$ and subsequent internal conversion, the absorption near time $t=0$ rapidly increases such as expected from Fig. 2. The subsequent decay of the signal is caused by CET to the bath. In agreement with our earlier studies, ${ }^{31-33}$ the energy loss of azulene is exponential and of the form $\langle E(t)\rangle \approx E_{0} \exp \left(-t / \tau_{\mathrm{CET}}\right)$ where $E_{0}$ is the photon energy of the excitation pulse and $\tau_{\mathrm{CET}}$ is a phenomenological cooling time constant. To model the experimental traces, $\langle E(t)\rangle$ was convoluted with the internal conversion process (time constant $\tau_{\mathrm{ic}}$ ) and a Gaussian shaped instrument response function of $\Delta \tau=0.2 \mathrm{ps}$ (full width at half maximum) representing the width of the cross correlation of pump and probe pulses, i.e.,

$$
\begin{aligned}
S(t) \propto & \int_{0}^{\infty} d \tau^{\prime} \int_{0}^{\tau^{\prime}} d \tau\langle E(\tau)\rangle \exp \left(-\frac{\tau^{\prime}-\tau}{\tau_{\text {ic }}}\right) \\
& \times \exp \left[-\left(\frac{t-\tau^{\prime}}{\Delta \tau}\right)^{2} 4 \ln 2\right],
\end{aligned}
$$

and fitted to the data. The result for $S(t)$ with $\tau_{\mathrm{CET}}=95 \mathrm{ps}$ is shown in Fig. 4 by the solid line. The CET time agrees with previous measurements of azulene collisional energy transfer in xenon. ${ }^{31}$ The error of $\tau_{\mathrm{CET}}$ is estimated to be $10 \%$.

The energy loss of the azulene side of the bridged compounds proceeds qualitatively different. This is shown in Fig. 5 for compound IV dissolved in xenon at 994 bar. Under these conditions the solvatochromic shift of the azulene $S_{3}$ band in IV with respect to bare azulene in the gas phases is $17 \mathrm{~nm}$. Therefore, in this case a probe wavelength of $307 \mathrm{~nm}$ guarantees that the presented signal is proportional to the energy-time profile of the azulene chromophore. Instead of a simple exponential decay, we observe two separate stages of energy loss. As in our previous study ${ }^{29}$ we attribute the faster component on the 5 ps time scale to intramolecular vibrational energy loss of the azulene chromophore through the bridge to the anthracene side. Intermolecular CET to the surrounding xenon bath then follows on a 30-40 ps time scale. Over the investigated xenon density range of $\rho_{\mathrm{Xe}}$ 


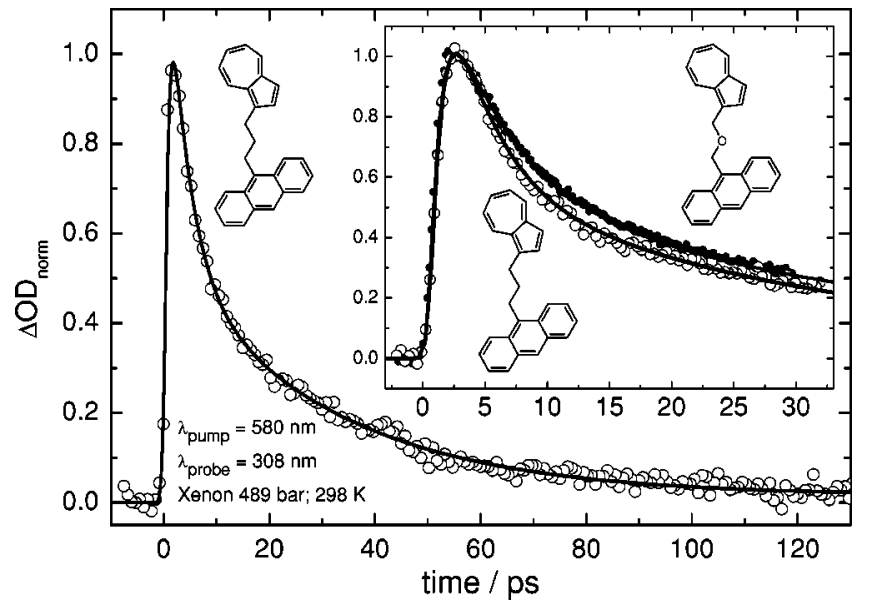

FIG. 6. Absorption-time profile of II in xenon at 489 bar and $298 \mathrm{~K}$ probing the vibrational energy content in the azulene chromophore. The insert shows a comparison with compound IV (filled circles) from Fig. 5 [full lines, fits assuming biexponential energy decays of Eq. (3) with amplitude ratios $A_{r}$ $=E_{\mathrm{IVR}} / E_{\mathrm{CET}}($ see text and Table I $\left.)\right]$.

$=2.1-2.7 \mathrm{~g} / \mathrm{cm}^{-8}$, no change of the IVR time constant of about 5 ps was observed, whereas the CET time scales varied inversely proportional to $\rho_{\mathrm{Xe}}$. The latter observation is in accord with our extensive studies on the density dependence of CET for azulene in xenon and in other supercritical fluids. ${ }^{31-33,41,42}$ Note, however, that the absolute CET rate of IV in xenon is more than two times faster than for bare azulene in xenon (see Fig. 4).

In Fig. 6 absorption signals for compound II in xenon at 489 bar are shown. As before, the energy loss of the azulene side is observed. In the insert, IVR of the compounds II and IV are compared. The plot shows that IVR is slightly faster for the aliphatic bridge than for the ether bridge. The amplitude ratios of IVR to CET are similar for both molecules. The latter can be rationalized by the fact that both molecules are of similar size and nearly the same amounts of energy leave the azulene side before intramolecular equilibrium is established. The CET rates of both molecules in xenon turn out to be approximately equal. The same behavior is observed when IVR and CET time scales for the compounds III and $\mathbf{V}$ are compared.

In our recent study of azulene-aryl compounds with bridges up to three methylene groups we found that IVR times linearly depend on the chain length. ${ }^{29}$ In contrast to this, for longer chains we now observe that the time constants level off and become independent of chain length. This is shown in Fig. 7 where signals for the compounds IV and $\mathbf{V}$ are compared in the insert. Although the bridge lengths of the compounds IV and $\mathbf{V}$ differ by almost a factor of 2, the times needed for intramolecular energy equilibration are nearly the same. Only the amplitude of the IVR component for $\mathbf{V}$ is slightly larger than for IV. This, however, simply reflects the differing heat capacities of the two compounds: before microcanonical equilibrium is achieved, more energy after excitation has to leave the azulene side of $\mathbf{V}$ than in the case of compound IV.

The given interpretation of separable IVR and CET in Figs. 5-7 is supported by the fact that the same time scales

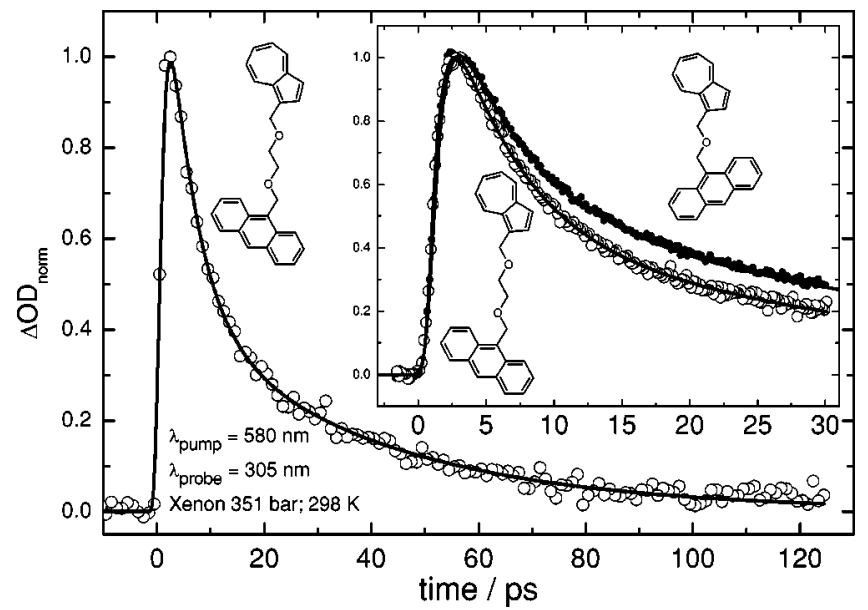

FIG. 7. Absorption-time profile of $\mathbf{V}$ in xenon at 351 bar and $298 \mathrm{~K}$ probing the vibrational energy content in the azulene chromophore. The insert shows a comparison with compound IV (filled circles) from Fig. 5 [full lines, fits assuming biexponential energy decays of Eq. (3) with amplitude ratios $A_{r}$ $=E_{\mathrm{IVR}} / E_{\mathrm{CET}}($ see text and Table I)].

also appear when the vibrational energy of the anthracene side at the red edge of its $S_{1}$ absorption band is probed. In Fig. 8, corresponding signals are shown for compound II dissolved in $\mathrm{C}_{2} \mathrm{Cl}_{3} \mathrm{~F}_{3}$ (the insert enlarges the early time dynamics). The probe wavelength was $\lambda_{\mathrm{pr}}=404 \mathrm{~nm}$. At time zero the signal in the insert of Fig. 8 shows a strong and sharp "coherent artifact" with a width of 200 fs arising from nonresonant absorption during the overlap of pump and probe pulse in the sample. Then, indeed a picosecond rise is observed followed by a much slower decay. Fitting a model function of the form $A\left\{\exp \left[-\left(t-t_{0}\right) / \tau_{\text {decay }}\right]-\exp [-(t\right.$ $\left.\left.\left.-t_{0}\right) / \tau_{\text {rise }}\right]\right\}$ to the signal, such as shown by the dashed line in the insert of Fig. 8, leads to $\tau_{\text {rise }} \cong 4 \mathrm{ps}, \tau_{\text {decay }} \cong 18 \mathrm{ps}$, and $t_{0} \cong-0.7 \mathrm{ps}$. $\tau_{\text {rise }}$ and $\tau_{\text {decay }}$ only approximately represent IVR and CET times, respectively, because the $\epsilon(\langle E\rangle)$ calibration curves for anthracene are nonlinear (see Fig. 4 in Ref.

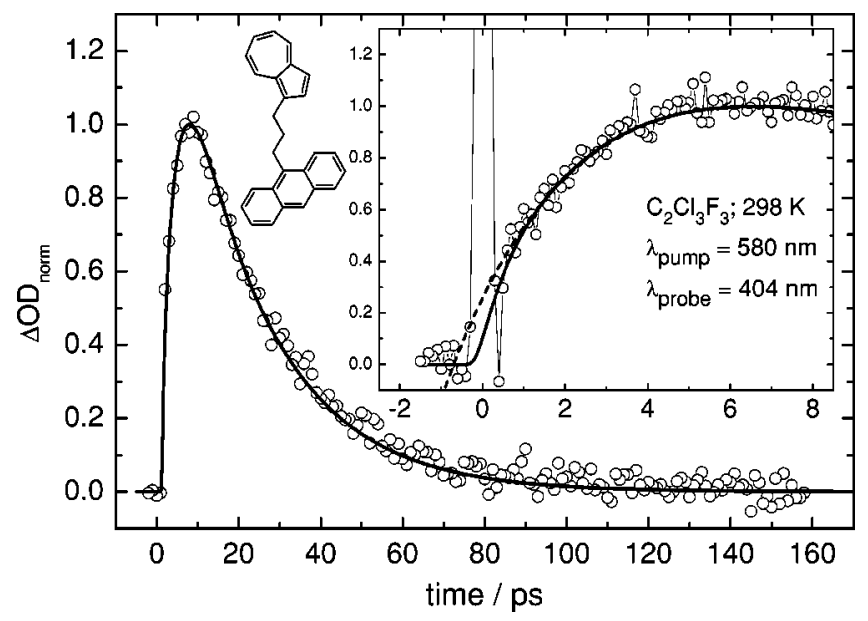

FIG. 8. Absorption-time profile of $\mathbf{I I}$ in $\mathrm{C}_{2} \mathrm{Cl}_{3} \mathrm{~F}_{3}$ probing the vibrational energy in the anthracene chromophore. The insert enlarges the short time dynamics and shows a sharp "coherence artifact" at $t=0$ [dashed line, fit with monoexponential rise and decay; full lines, assuming energy evolution according to Eq. (4) with amplitude ratios $A_{r}^{\prime}=E_{\mathrm{IVR}}^{\prime} / E_{\mathrm{CET}}^{\prime}$ (see text and Table I)]. 
29). A closer comparison between the dashed line and the signal at around $t=0$ suggests that, prior to the $4 \mathrm{ps}$ rise, there must be a much faster component transferring energy to the anthracene side within a time which we cannot resolve since it is hidden by the coherent artifact. We will discuss this point in more detail in the following section.

\section{B. Harmonic energy flow}

Figure 8 suggests that a significant amount of energy enters the anthracene side on a subpicosecond time scale. We attribute this process to a "harmonic energy flux" by dephasing of the projection of normal modes on the atomic displacements and momenta of the considered compounds which is connected with the IVR process in the azulene chromophore itself. This is because a random local excitation of only the azulene side can always be approximated by a superposition of normal modes (with particular amplitudes and phases). Each of these normal modes is more or less delocalized over the whole molecule. Since the normal modes develop in time with different phase velocities any local excitation inevitably changes. After selective azulene excitation this process leads to energy loss at the azulene side and energy gain in the chain and at the anthracene side. Thus, the mechanism responsible for the fast increase of the anthracene energy in Fig. 8 can be understood on the basis of a normalmode harmonic oscillator Hamiltonian once the energy is redistributed at the azulene side.

In order to estimate the amount of energy transferred and the characteristic time for this harmonic energy flux, we performed classical simulations using normal modes of azulene and of the bridged compounds which we determined by DFT calculations (the details will be presented in a subsequent paper ${ }^{43}$ ). The basic assumption for these simulations is that IVR inside azulene is much faster than IVR through the bridge such that a statistical energy distribution in azulene directly after laser excitation and subsequent internal conversion can be assumed. We start our simulation with a configuration of the whole molecule, say compound II, where the population and thus the amplitude $Q_{0, \alpha}^{\text {II }}$ of each normal mode $\alpha(1 \leqslant \alpha \leqslant 3 N-6 ; N$ is the number of atoms in the molecule) is sampled from a quantum statistical canonical distribution at room temperature. Since we consider a classical harmonic system, this gives us normal modes which evolve in time like $Q_{\alpha}^{\text {II }}(t)=Q_{0, \alpha}^{\text {II }} \cos \left(\omega_{\alpha} t+\phi_{\alpha}\right)$ with a randomly chosen phase $\phi_{\alpha}$. Transformation of the initial configuration to Cartesian coordinates yields coordinates $\mathbf{r}_{i}$ and velocities $\mathbf{u}_{i}$ of all atoms $i$ in II. For the excitation, we consider only the azulene side of II by projecting the corresponding atom coordinates and velocities onto the normal modes of the azulene molecule. This gives us normal coordinates $Q_{\beta}^{\mathrm{Az}}$ and velocities $\dot{Q}_{\beta}^{\mathrm{Az}}$ of the azulene part $(1 \leqslant \beta \leqslant 3 N-6 ; N$ now is the number of atoms in the azulene part). Subsequently, at $t=0$, the energy of the azulene side is increased by the photon energy $E_{0}$ of the laser excitation pulse which is distributed over the azulene normal modes according to a quantum statistical microcanonical distribution. The resulting excess energy for each azulene normal mode is transferred into kinetic energy by increasing $\dot{Q}_{\beta}^{\mathrm{Az}}$. Transformation back to Car-

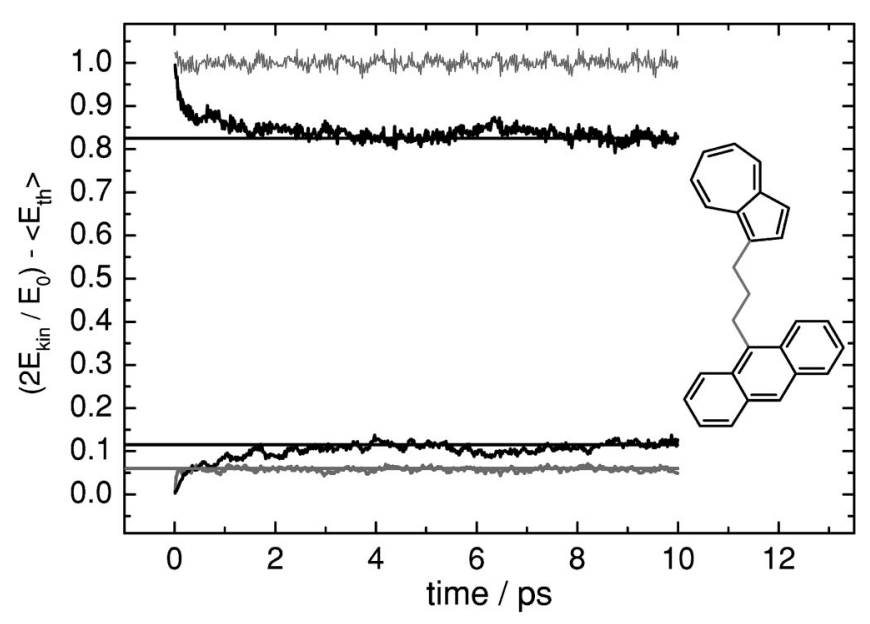

FIG. 9. Harmonic energy flow in compound II. Changes of the kinetic energy in different parts of the molecule are recorded during the propagation of normal modes after local excitation of the azulene chromophore (upper black line, energy in the azulene part; lower black line, in the anthracene part; lower gray line, in the chain; upper gray line, the sum of all contributions).

tesian coordinates gives $\mathbf{r}_{i}$ and $\mathbf{u}_{i}$ of all atoms $i$ in II where the velocities of the azulene atoms have been increased according to the excitation procedure. Finally, this configuration is projected onto the normal modes $Q_{\alpha}^{\mathbf{I I}}(t)$ of $\mathbf{I I}$, giving new amplitudes $Q_{0, \alpha}^{\text {II }}$ which then are propagated in time. At each time step, transformation to Cartesian coordinates allows to calculate the energies $E_{\text {kin }}$ of azulene, anthracene, and the chain. After averaging over several thousand initial configurations we obtain $E_{\text {kin }}(t)$ curves such as exemplarily shown in Fig. 9. The upper black curve represents the relative change of the azulene kinetic energy which after excitation drops to $\sim 82 \%$ within 300 fs. At the same time the energies of the chain (lower gray line) and the anthracene side (lower black line) are increased by $6 \%$ and $12 \%$ of the photon energy, respectively. Note that these energy changes are not caused by IVR because, in the described procedure, simply normal modes are propagated with constant amplitudes $Q_{0, \alpha}^{\text {II }}$. At $t=0$ in Fig. 9, the phases of all $Q_{\alpha}^{\text {II }}$ are such that the superposition of the normal modes leads to large displacements and momenta of the azulene atoms whereas the rest of the molecule remains unexcited. With increasing time the $Q_{\alpha}^{\text {II }}$ dephase and $18 \%$ of the excitation energy leaves the azulene side and flows into the chain and to the anthracene side. Our calculations show that the amount of energy leaving the azulene part is quite insensitive to the chain length. ${ }^{43}$ The time scale of 300 fs for the harmonic energy flow is faster than the azulene internal conversion process. Therefore, it cannot be resolved in our experiment. The amount of energy transferred, however, is in complete agreement with Fig. 8 and also with Figs. 5-7 considering the energy balance derived from the heat capacities of the chromophores (see the following section).

\section{Energy balance of intramolecular and intermolecular vibrational energy transfer}

In order to quantify the vibrational energy transported during intramolecular equilibration in the bridged azulene- 


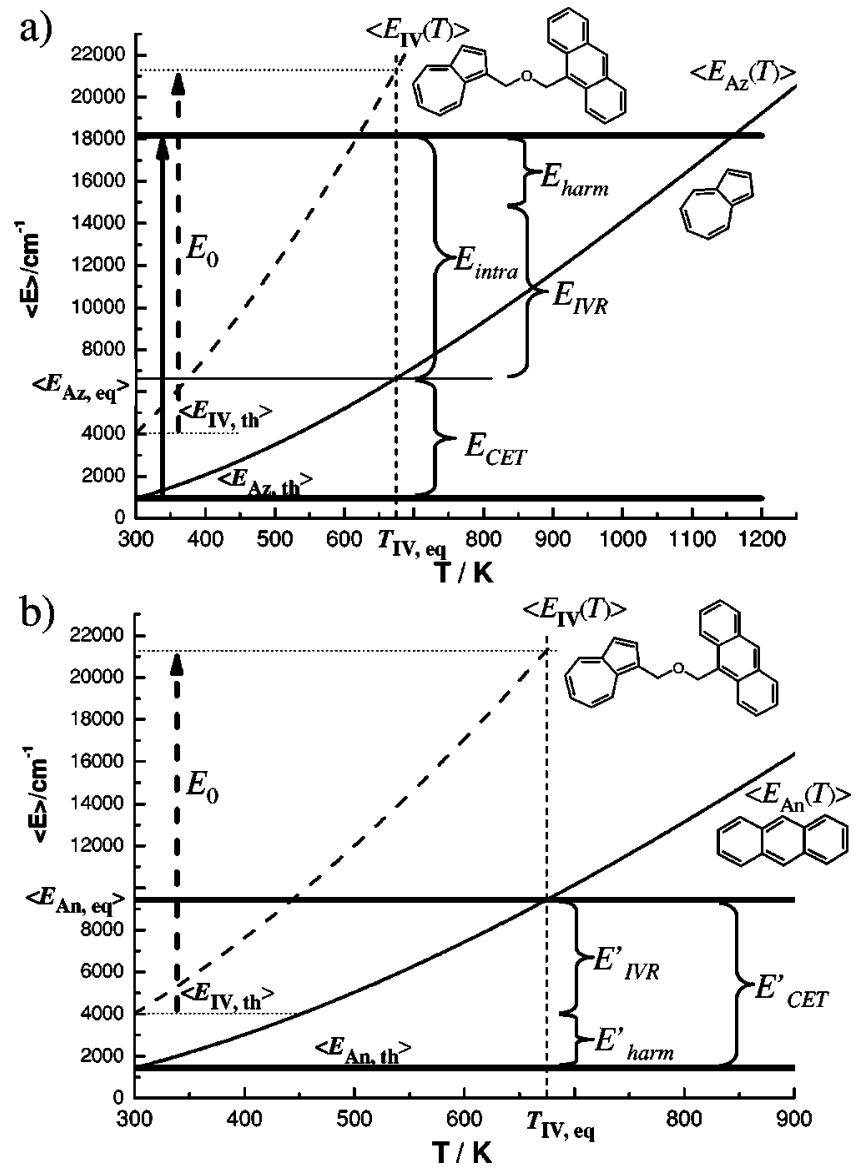

FIG. 10. Vibrational energies as a function of temperature for azulene and IV (a), anthracene and IV (b). From these diagrams the energy amplitudes of Eqs. (3) and (4) are derived (see text).

anthracene compounds the relative heat capacities of the two chromophores have to be considered. This finally allows to specify the amplitude ratio $A_{r}$ of the fast (IVR) and slow (CET) decaying components of the azulene signals in Figs. 5-7. The procedure to determine $A_{r}$ for compound IV is demonstrated in Fig. 10. At first, energy-temperature relations were calculated from the $s$ normal-mode frequencies $\nu_{i}$ of the considered molecules via

$$
\langle E(T)\rangle=\sum_{i=1}^{s} \frac{h \nu_{i}}{\exp \left(h \nu_{i} / k_{B} T\right)-1}
$$

(frequencies were determined from DFT calculations). The results $\left\langle E_{\mathbf{I V}}(T)\right\rangle$ for $\mathbf{I V}$ and $\left\langle E_{\mathrm{Az}}(T)\right\rangle$ for the azulene part only are shown in Fig. 10(a) by the dashed and full lines, respectively. The lower full horizontal line represents the average thermal energy $\left\langle E_{\mathrm{Az}, \mathrm{th}}\right\rangle=940 \mathrm{~cm}^{-1}$ of the azulene side at the beginning of the experiment. After laser excitation, the azulene energy increases to $18180 \mathrm{~cm}^{-1}$ as indicated by the full arrow and the upper full horizontal line. When this energy equilibrates over the whole molecule $\mathbf{I V}$, only part of it remains at the azulene side. This part can be calculated by means of the $\left\langle E_{\mathbf{I V}}(T)\right\rangle$ curve of compound IV. After laser excitation, the total vibrational energy in IV is given by its thermal energy $\left\langle E_{\mathbf{I V}, \text { th }}\right\rangle=4040 \mathrm{~cm}^{-1}$ plus the photon energy $E_{0}$ (dashed arrow). When the energy is equilibrated over the whole molecule, this corresponds to a vibrational temperature of $T_{\mathrm{IV} \text {, eq }}=670 \mathrm{~K}$. The $\left\langle E_{\mathrm{Az}}(T)\right\rangle$ curve shows that at this temperature the remaining energy at the azulene side is about $\left\langle E_{\mathrm{Az}, \mathrm{eq}}\right\rangle=6650 \mathrm{~cm}^{-1}$. The difference $\left\langle E_{\mathrm{Az}, \mathrm{eq}}\right\rangle-\left\langle E_{\mathrm{Az}, \mathrm{th}}\right\rangle$ is finally transferred to the surrounding by CET. Therefore, the amplitudes of intramolecular and intermolecular energy transfer components are given by $E_{\text {intra }}$ and $E_{\mathrm{CET}}$ as indicated in Fig. 10(a).

In the preceding section we demonstrated that, after randomization at the azulene side, $18 \%$ of the excitation energy $E_{0}$ is directly transferred to the chain and to the anthracene side by harmonic energy flow within 300 fs. The difference $E_{\mathrm{IVR}}=E_{\text {intra }}-E_{\text {harm }}=E_{\text {intra }}-0.18 E_{0}$ has to be redistributed via IVR to reach microcanonical equilibrium. With respect to internal conversion, the harmonic energy flow is much too fast to be resolved when the energy content of the azulene side is probed. Therefore, we assign the fast and slow decaying components observed in Figs. 5-7 to IVR and CET, respectively, with amplitude ratios given by $A_{r}$ $=E_{\mathrm{IVR}} / E_{\mathrm{CET}}$. In fact, biexponential fits of the form

$$
E_{\mathrm{An}}(t) \propto A_{r} \exp \left(-t / \tau_{\mathrm{IVR}}\right)+\exp \left(-t / \tau_{\mathrm{CET}}\right)
$$

[convoluted with the internal conversion process and the instrument response function according to Eq. (1)] are in excellent agreement with the signals as shown by the full lines in Figs. 5-7. The energies $E_{\mathrm{IVR}}$ and $E_{\mathrm{CET}}$ calculated from the normal modes, as well as the parameters $\tau_{\mathrm{IVR}}$ and $\tau_{\mathrm{CET}}$ fitted to the curves, are summarized in Table I. Likewise, in Fig. 5 we present a biexponential fit (dashed line) where the amplitude ratio of fast and slow decaying component was fixed to

\begin{tabular}{|c|c|c|c|c|c|c|}
\hline Compound & $\mathbf{I}$ & II & III & IV & $\mathbf{V}$ & VI \\
\hline \multicolumn{7}{|l|}{ Eq. (3) } \\
\hline$E_{\mathrm{IVR}} / \mathrm{cm}^{-1}$ & 7980 & 8736 & 9940 & 8950 & 9780 & 9040 \\
\hline$E_{\mathrm{CET}} / \mathrm{cm}^{-1}$ & 5810 & 5570 & 4710 & 5700 & 4870 & 5620 \\
\hline $\begin{array}{l}\text { Eq. (4) } \\
E_{\mathrm{IVR}}^{\prime} / \mathrm{cm}^{-1} \\
E_{\mathrm{CFT}}^{\prime} / \mathrm{cm}^{-1}\end{array}$ & & $\begin{array}{l}5900 \\
7800\end{array}$ & & & & \\
\hline$\tau_{\mathrm{IVR}} / \mathrm{ps}$ & $3.7 \pm 0.4$ & $3.7 \pm 0.3$ & $4.2 \pm 0.6$ & $5.0 \pm 0.4$ & $5.1 \pm 0.4$ & $5.1 \pm 0.5$ \\
\hline$\tau_{\mathrm{CET}} / \mathrm{ps}$ & $\mathrm{C}_{2} \mathrm{Cl}_{3} \mathrm{~F}_{3}: 23 \pm 2$ & $\begin{array}{c}\mathrm{C}_{2} \mathrm{Cl}_{3} \mathrm{~F}_{3}: 20 \pm 2 \\
\mathrm{Xe}, 489 \text { bar: } 35 \pm 3\end{array}$ & Xe, 358 bar: $40 \pm 5$ & $\begin{array}{l}\text { Xe, } 288 \text { bar: } 40 \pm 3 \\
\text { Xe, } 994 \text { bar: } 34 \pm 3\end{array}$ & $\begin{array}{l}\text { Xe, } 351 \text { bar: } 34 \pm 3 \\
\text { Xe, } 976 \text { bar: } 29 \pm 3\end{array}$ & $\mathrm{CH}_{2} \mathrm{Cl}_{2}: 14 \pm 2$ \\
\hline
\end{tabular}

TABLE I. Parameters for Eqs. (3) and (4) to describe intramolecular and intermolecular vibrational energy transfer in compounds I-VI. 
$A_{r}=E_{\text {intra }} / E_{\mathrm{CET}}$, thereby identifying $E_{\mathrm{IVR}}$ with $E_{\mathrm{intra}}$ and neglecting $E_{\text {harm }}$. The deviations from the measured curve clearly indicate that the amplitude of the IVR component is too large which supports our interpretation that, prior to IVR, a significant amount of energy leaves the azulene side on a subpicosecond time scale due to harmonic energy flow.

The energy of the anthracene side may be described by

$$
\begin{aligned}
E_{\mathrm{An}}(t)= & E_{\mathrm{CET}}^{\prime}\left\{\exp \left(-t / \tau_{\mathrm{CET}}\right)-\left[A_{r}^{\prime} \exp \left(-t / \tau_{\mathrm{IVR}}\right)\right.\right. \\
& \left.\left.+\left(1-A_{r}^{\prime}\right) \exp \left(-t / \tau_{\text {harm }}\right)\right]\right\},
\end{aligned}
$$

where the parameters $E_{\mathrm{CET}}^{\prime}$ and $A_{r}^{\prime}$ are derived from Fig. 10(b). Employing the $\left\langle E_{\mathrm{An}}(T)\right\rangle$ curve calculated from the anthracene normal modes and using the same arguments as for Fig. 10(a), it is easy to show that, after vibrational excitation and randomization, the energy of the anthracene side will rise from $\left\langle E_{\mathrm{An}, \mathrm{th}}\right\rangle$ (the thermal energy at $300 \mathrm{~K}$ ) to $\left\langle E_{\mathrm{An}, \mathrm{eq}}\right\rangle$ (the energy at microcanonical equilibrium). This intramolecular equilibration proceeds in two steps: by fast harmonic energy flow increasing the anthracene energy by $E_{\text {harm }}^{\prime}=0.12 E_{0}$ (see Fig. 9) which then is followed by the slower IVR process transferring $E_{\mathrm{IVR}}^{\prime}=\left(\left\langle E_{\mathrm{An}, \text { eq }}\right\rangle-\left\langle E_{\mathrm{An}}\right\rangle\right)$ $-E_{\text {harm }}^{\prime}$. All the energy $E_{\mathrm{CET}}^{\prime}=E_{\text {harm }}^{\prime}+E_{\mathrm{IVR}}^{\prime}$ is finally dissipated into the solvent. Therefore, the amplitude ratio in Eq. (4) is given by $A_{r}^{\prime}=E_{\mathrm{IVR}}^{\prime} / E_{\mathrm{CET}}^{\prime}$. Since the $\epsilon(\langle E\rangle)$ calibration curves of anthracene are nonlinear, ${ }^{29}$ the signal in Fig. 8 is not simply proportional to energy. Taking into account the solvatochromic shift of the anthracene $S_{1}$ band of II with respect to pure anthracene in the gas phase, the $E_{\mathrm{An}}(t)$ curves were transformed into absorption changes $\Delta \epsilon(t)$ using the $\epsilon(\langle E\rangle)$ calibration curves of Fig. 4 from Ref. 29. Finally, $\Delta \epsilon(t)$ was convoluted with the internal conversion process and the instrument response function and fitted to the signal by adjusting $\tau_{\mathrm{IVR}}$ and $\tau_{\mathrm{CET}}$ in Eq. (4). The result is shown by the full line in Fig. 8. Considering the fact that $E_{\mathrm{CET}}^{\prime}$ and $A_{r}^{\prime}$ were fixed parameters derived from the vibrational frequencies of the molecules, the agreement with the measured curve is very good. The quality of the fit is insensitive to $\tau_{\text {harm }}$ as long its value is below 0.4 ps because the initial fast rise is limited essentially by the internal conversion time.

\section{DISCUSSION}

So far our analysis shows that, after $S_{1}$ excitation of the azulene chromophore and subsequent internal conversion, the energy dissipation proceeds in three steps. In a first period being faster than the internal conversion process itself, the vibrational energy is randomized at the azulene side. This establishes a "vibrational temperature" and allows to use thermal spectra for calibrating absorption signals against internal energies. Energy redistribution in the azulene chromophore is accompanied by a considerable harmonic flow of vibrational energy into the bridge and into the anthracene part. Since these initial processes occur within several hundreds of femtoseconds, their temporal evolution cannot be followed in this work. The presence of the harmonic energy flow, however, shows up in the signal of Fig. 8 by a fast increase of the anthracene energy at around $t=0$; it also results from the analysis of the azulene energy decay consid-

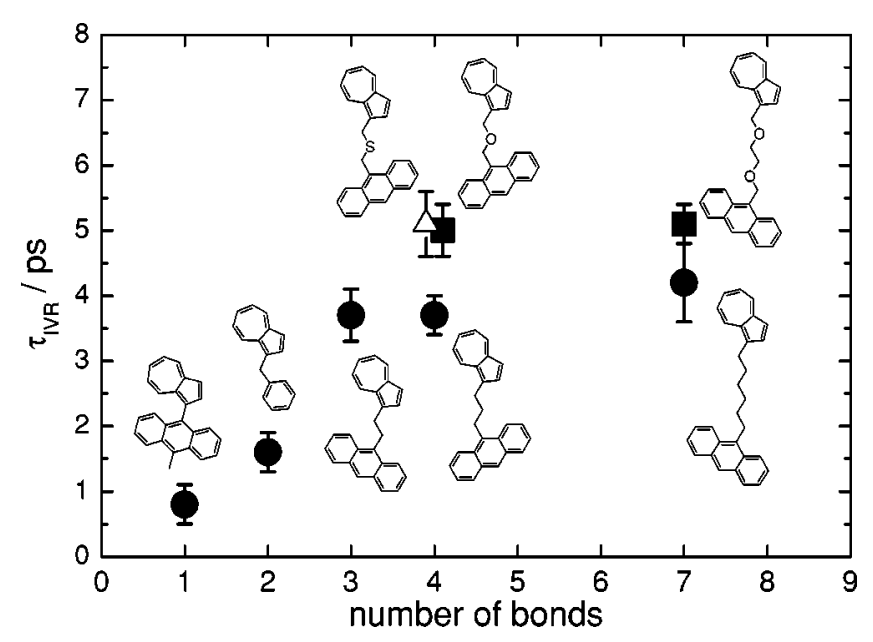

FIG. 11. IVR times vs the number of bonds in the bridge connecting the chromophores (full circles, aliphatic chains; full squares, ethers; open triangle, thio ether; data for short bridges with $n=1,2$ were taken from our previous study. ${ }^{29}$

ering the heat capacities of the molecules such as discussed in the preceding section. After this initial period, the energy flux is dominated by IVR within the whole molecule on a picosecond time scale until intramolecular equilibrium is reached. Finally collisional energy transfer to the surrounding medium, taking several tens of picoseconds, cools down the excited molecules completely.

It should be mentioned that related experiments by Okazaki et al. ${ }^{44}$ with azulene- $\left(\mathrm{CH}_{2}\right)_{n}$-coumarin $151(n=1,3)$ in solution were aimed at studying the temperature distribution of the solvent around the excited azulene molecule during the CET process by using coumarin 151 as a thermometer located at a well defined distance from the heater molecule. Our experiments, however, clearly show that this approach cannot work with this type of molecules because IVR through the bridge is much more efficient than energy flow from azulene through the solvent to the acceptor chromophore.

In the following we discuss the dependence on the length of the bridge of the IVR time constant describing the energy flow from the azulene to the anthracene side. In Fig. 11 the experimental IVR times $\tau_{\mathrm{IVR}}$ are plotted vs the number of chemical bonds $n$ of the bridge. The plot also includes some data from our previous study. ${ }^{29}$ For short chains, a nearly linear increase of $\tau_{\mathrm{IVR}}$ with the length of the bridge is observed whereas at $n \geqslant 4$ the IVR time levels off when $\tau_{\text {IVR }}$ for II and III as well as for IV and V are compared. Note that the IVR time constants for the aliphatic chains are slightly shorter than for the (thio)ethers (compare compounds I, IV, VI, and III, V, respectively). The linear increase of $\tau_{\text {IVR }}$ with chain length for $n \leqslant 4$ is consistent with a recent IVR study on linear alcohols, where the $\mathrm{OH}$-stretch vibration was excited and the energy flow to the terminal $\mathrm{CH}_{3}$ group was monitored. ${ }^{16}$ For the alcohols ethanol to 1-butanol the corresponding energy transfer time increased by about 0.4 ps for each additional methylene group between $\mathrm{OH}$ and $\mathrm{CH}_{3}$.

In our previous paper ${ }^{29}$ we used a phonon diffusion model analogous to Fourier's law of heat conduction in order 
to describe the energy transport through short bridges with $n \leqslant 4$,

$$
\frac{d E}{d t}=-\kappa \frac{\partial T}{\partial x},
$$

where $\kappa$ is the thermal conductivity of the molecular chain aligned in $x$ direction and $T$ is the temperature. Since, for short bridges, $\tau_{\text {IVR }}$ scales with the length of the bridge, it was possible to derive a "molecular energy conductivity" of a single $\mathrm{C}-\mathrm{C}$ bond (with length $l)$ of $\kappa_{\mathrm{CC}}=\kappa / l=(10$ $\pm 1) \mathrm{cm}^{-1} \mathrm{~K}^{-1} \mathrm{ps}^{-1}$ which is constant for all compounds with $n \leqslant 4$. Figure 11 , however, shows that, for $n \geqslant 4$, the time for intramolecular equilibration becomes nearly constant. This happens although the temperature gradient between the chromophores decreases with increasing chain length. If a heat conduction model is used to reproduce this behavior, consequently, $\kappa_{\mathrm{CC}}$ has to increase proportional to the length of the bridge. This result seems to be counterintuitive and signals that Eq. (5) only represents a phenomenological description which may not be adequate for a characterization of the vibrational energy flow on an atomic scale.

\section{A. Comparison with molecular dynamics simulations}

In order to examine the intramolecular vibrational energy flow in bridged azulene-anthracene compounds in more detail, we performed classical molecular dynamics (MD) calculations. ${ }^{43}$ Naturally this approach suffers from neglecting the quantum nature of molecular vibrations. Moreover, the generally used force fields describing the intramolecular potential and anharmonicities causing couplings between the vibrational modes are highly artificial. Therefore, from a simulation one cannot expect quantitative agreement with the experiment. Nevertheless, it appears attractive to investigate whether similar dependences of the IVR time constants on the length of the chain are found in MD models as in the experiment. We, therefore, did classical nonequilibrium MD simulations with molecules of the type azulene$\left(\mathrm{CH}_{2}\right)_{m}$-anthracene with $m=0-10$. Details of these calculations, including the molecular force fields employed and the specific excitation procedure, are described elsewhere. ${ }^{43}$ Briefly, for a particular molecule, the simulation started by sampling configurations (atom coordinates and velocities) from a canonical distribution at $300 \mathrm{~K}$. Excitation was achieved by scaling the atom velocities of the azulene part such that the overall vibrational energy increase corresponded to the photon energy $E_{0}$. Then trajectories were followed for $150 \mathrm{ps}$ within the NVE ensemble. At each time step the kinetic vibrational energies $E_{\text {kin }}$ of the azulene and anthracene part as well as the methylene groups of the chain were recorded. Averaging was accomplished over 200 statistically independent trajectories.

The results of these simulations can be summarized as follows. The intramolecular energy flow does not depend on whether the calculations are done for the isolated molecule or for the azulene-anthracene compound dissolved in a bath of xenon. Therefore, most of the calculations were done for the isolated molecule. In Fig. 12 a typical result of a MD simulation is shown for the compound III. From the kinetic vibrational energies of the atoms in III the temperatures of

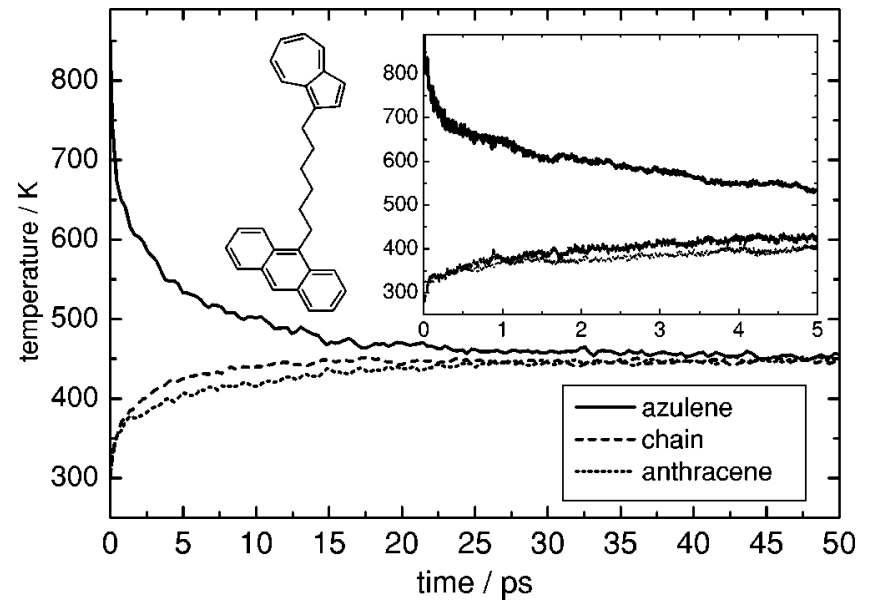

FIG. 12. Molecular dynamics simulation of intramolecular vibrational energy redistribution in isolated III (the vibrational temperatures of different parts of the molecule are plotted vs time; the insert enlarges the short time dynamics).

different parts of the molecule were calculated and plotted vs time. In accord with the experimental observation intramolecular equilibration proceeds in two steps. There is a fast subpicosecond component caused by harmonic energy flow followed by the IVR process taking several picoseconds (for calculations in xenon a third time scale related to CET is observed; the corresponding time constants are about $50 \%$ larger than the experimental values). The harmonic energy flow is most clearly seen for the azulene temperature which within the first 300 fs decays from approximately 800 to 700 $\mathrm{K}$ (see insert in Fig. 12). This corresponds to a loss of $20 \%$ of the excitation energy and is in complete agreement with the analysis given in Sec. III B and with Fig. 9 where for compound II the azulene chromophore lost $18 \%$ of the excitation energy as a result of harmonic energy flow. Subsequently, in Fig. 12, intramolecular energy equilibration takes place leading to an equilibrium temperature of $450 \mathrm{~K}$. The time constant for this process is $\tau_{\mathrm{IVR}}=(7 \pm 1) \mathrm{ps}$. For $m$ $>1$, in general, IVR can be described by single exponential curves. On average, the calculated time constants $\tau_{\text {IVR }}$ were about 50\% larger than the experimental values. Most interestingly, however, the dependence of $\tau_{\text {IVR }}$ on the length of the bridge showed the same trends as found in the experiment. In particular, for $n>4$ the time for intramolecular energy redistribution became constant and independent of the chain length such as demonstrated in Fig. 13. This observation suggests that in both, experiment and simulation, similar mechanisms dominate the energy exchange through the molecular bridge. A closer inspection of the MD results, therefore, allows to identify the operating mechanisms.

In Fig. 14, the calculated temperature profiles from the azulene side (left) through the bridge to the anthracene side (right) in compound III are plotted while the energy equilibrates. The graphs show that during the whole process a strong temperature gradient at the connection of the azulene molecule to the methylene chain persists whereas in the chain this gradient vanishes. For molecules with shorter chains, we observe strong temperature gradients not only at the azulene but also at the anthracene side, indicating that the 


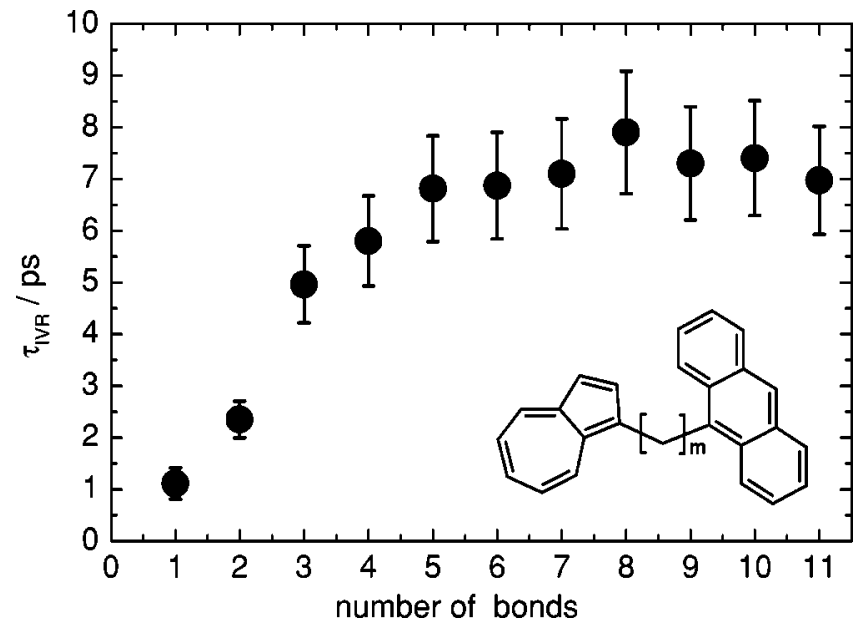

FIG. 13. IVR times vs the number of bonds in the chain for azulene$\left(\mathrm{CH}_{2}\right)_{m}$-anthracene as derived from molecular dynamics simulations (compare with Fig. 11).

coupling of the chromophores to the bridge is limiting the energy flux. In the chain the energy transport obviously proceeds much faster and, therefore, the time needed to distribute the vibrational energy over the whole molecule with increasing chain length remains the same for $n>4$. The strong temperature gradient in Fig. 13 extends over two to three bonds which might be the reason that, for shorter bridges, $\tau_{\text {IVR }}$ is proportional to the chain length.

At this point it is instructive to come back to the heat conduction model of Eq. (5) and ask for the prerequisites of diffusive energy transport. Within kinetic theory, vibrational energy in dielectric crystals is transferred via phonons which propagate through the lattice like quasiparticles. Due to "collisions" arising from nonlinear interactions or disorder in the lattice, new phonons are formed. According to Peierls ${ }^{45}$ these collisions can be classified into two groups: those where phonon momentum is conserved (the normal processes) and those where the initial and final momenta differ by a nonzero reciprocal lattice vector (Umklapp or $U$ processes). Umklapp processes are essential for diffusive energy transport: the

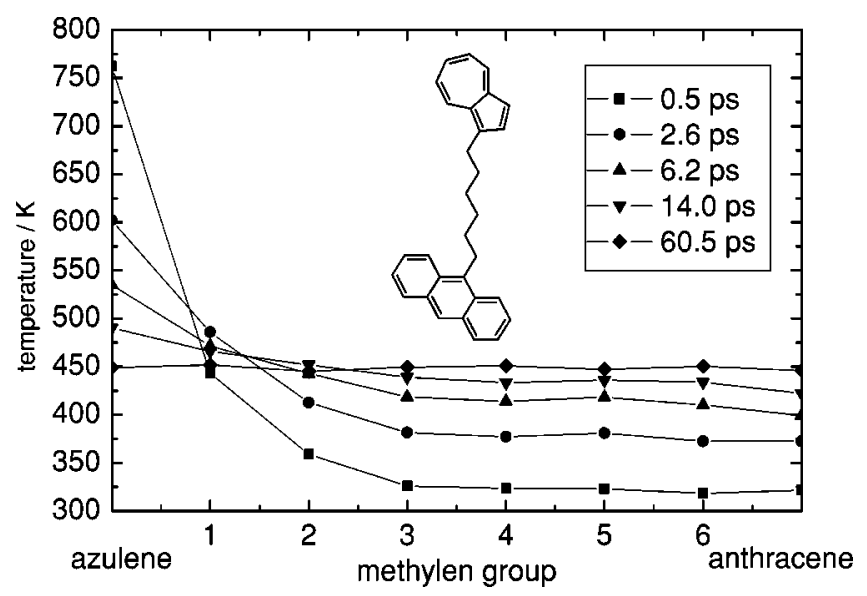

FIG. 14. Molecular dynamics simulation of intramolecular vibrational energy redistribution in isolated III [temperature profiles in the bridge including the azulene (left) and the anthracene chromophore (right) are plotted at various times]. thermal conductivity is given by $\kappa^{\propto} c_{v}\langle v\rangle l$, where $c_{v}$ is the specific heat at constant volume, and $\langle v\rangle$ and $l$ are the average velocity and the mean free path of phonons before they undergo an Umklapp process, respectively. If the dimensions of the dielectric medium are smaller than $l$, the Fourier modes freely propagate through the lattice, thus contributing to ballistic rather than diffusive energy transport. In this case the flux no longer is proportional to the gradient but to the temperature difference between the heat baths

$$
\frac{d E}{d t}=-\kappa^{\prime}\left(T_{2}-T_{1}\right)
$$

where $\kappa^{\prime}$ depends on the coupling strength of the considered medium to the reservoirs. Theoretical work on onedimensional harmonic lattices shows that the temperature profile in the medium is essentially flat in the ballistic regime. ${ }^{17,46}$

The results of our experiments and MD simulations on IVR in bridged azulene-anthracene compounds, thus, can consistently be explained by assuming ballistic energy transport in the chain connecting both chromophores. Since, for $n \geqslant 4, \tau_{\mathrm{IVR}}$ is essentially constant in Fig. 11, the apparent thermal conductivity of the chain seems to increase proportional to the length of the chain when using Eq. (5) instead of Eq. (6). Figure 14 shows that the temperature gradient in the bridge vanishes which also indicates free energy transport and absence of diffusive scattering events.

\section{CONCLUSIONS}

In the present work we have investigated intramolecular vibrational energy flow through molecular chains which at each side are connected to a chromophore acting as a heat reservoir. At one side, azulene served as the energy donor being vibrationally excited by light absorption into the $S_{1}$ state and subsequent internal conversion back to the ground electronic state within less than $1 \mathrm{ps}$. At the other side, anthracene was the energy acceptor. The vibrational energy flow was followed by monitoring the vibrational temperatures of one or both of the chromophores using the hot band absorption technique. Two distinct regimes of intramolecular dynamics were identified: during a 300 fs period, $15 \%-20 \%$ of the excitation energy leaves the azulene chromophore by "harmonic energy flow" caused by dephasing of the projection of normal modes on the atomic displacements and momenta of the considered compounds. This process is intimately connected to the fast energy randomization within the azulene chromophore itself. Intramolecular energy flow towards a microcanonical equilibrium within the whole molecule is completed by IVR. The corresponding time constants $\tau_{\text {IVR }}$ vary between 1 and 5 ps. Interestingly, the IVR time constants for bridges consisting of more than four bonds become constant and independent of chain length. Detailed molecular dynamics simulation showed that the energy flux in these systems is limited by the coupling of the heat baths to the chain. Inside the bridge, phonons freely propagate which corresponds to ballistic transport. This finding is in agreement with theoretical investigations on heat conduction in one-dimensional dielectric lattices. 


\section{ACKNOWLEDGMENTS}

The authors are grateful to Jens Schimpfhauser and Jürgen Bienert for the preparation and purification of the investigated azulene-anthracene compounds. We also thank Bernd Abel, Günter Käb, and Jörg Schroeder for many helpful discussions. Financial support of this project by the Deutsche Forschungsgemeinschaft (SFB357 "Molekulare Mechanismen unimolekularer Prozesse") is gratefully acknowledged.

${ }^{1}$ D. J. Nesbitt and R. W. Field, J. Phys. Chem. 100, 12735 (1996).

${ }^{2}$ R. G. Gilbert and S. C. Smith, Theory of Unimolecular and Recombination Reactions (Blackwell Scientific, Oxford and Cambridge, Mass., 1990).

${ }^{3}$ T. Baer and W. L. Hase, Unimolecular Reaction Dynamics (Oxford, New York, 1996).

${ }^{4}$ M. Quack, Annu. Rev. Phys. Chem. 41, 839 (1990).

${ }^{5}$ K. K. Lehmann, G. Scoles, and B. H. Pate, Annu. Rev. Phys. Chem. 45, 241 (1994).

${ }^{6}$ A. Laubereau and W. Kaiser, Rev. Mod. Phys. 50, 607 (1978).

${ }^{7}$ G. Seifert, R. Zürl, and H. Graener, J. Phys. Chem. A 103, 10749 (1999).

${ }^{8}$ S. Woutersen, U. Emmerichs, and H. J. Bakker, Science 278, 658 (1997).

${ }^{9}$ M. M. Heckscher, L. Sheps, D. Bingemann, and F. F. Crim, J. Chem. Phys. 117, 8917 (2002).

${ }^{10}$ J. Assmann, A. Charvat, D. Schwarzer, C. Kappel, K. Luther, and B. Abel, J. Phys. Chem. A 106, 5197 (2002).

${ }^{11}$ R. von Benten, O. Link, B. Abel, and D. Schwarzer, J. Phys. Chem. A 108, 363 (2004)

${ }^{12}$ R. von Benten, A. Charvat, O. Link, B. Abel, and D. Schwarzer, Chem. Phys. Lett. 386, 325 (2004).

${ }^{13}$ J. D. Rynbrandt and B. S. Rabinovitch, J. Phys. Chem. 75, 2164 (1971).

${ }^{14}$ I. Oref and B. S. Rabinovitch, Acc. Chem. Res. 12, 166 (1979).

${ }^{15}$ S. P. Wrighley and B. S. Rabinovitch, Chem. Phys. Lett. 95, 363 (1983).

${ }^{16}$ Z. Wang, A. Pakoulev, and D. D. Dlott, Science 296, 2201 (2002).

${ }^{17}$ S. Lepri, R. Livi, and A. Politi, Phys. Rep. 377, 1 (2003).

${ }^{18}$ U. Sukowski, A. Seilmeier, T. Elsaesser, and S. F. Fischer, J. Chem. Phys. 93, 4094 (1990).

${ }^{19}$ S. Lepri, R. Livi, and A. Politi, Phys. Rev. Lett. 78, 1896 (1997).

${ }^{20}$ C. Giardina, R. Livi, A. Politi, and M. Vasalli, Phys. Rev. Lett. 84, 2144 (2000).

${ }^{21}$ B. Hu, B. Li, and H. Zhao, Phys. Rev. E 61, 3828 (2000).

${ }^{22}$ A. Dhar, Phys. Rev. Lett. 86, 5882 (2001).
${ }^{23}$ P. Grassberger, W. Nadler, and L. Yang, Phys. Rev. Lett. 89, 180601 (2002).

${ }^{24}$ D. M. Leitner and P. G. Wolynes, Phys. Rev. E 61, 2902 (2000).

${ }^{25}$ D. Neuhauser, R. Baer, and R. Kosloff, J. Chem. Phys. 118, 5729 (2003).

${ }^{26}$ D. Schwarzer, J. Troe, and J. Schroeder, Ber. Bunsenges. Phys. Chem. 95, 933 (1991).

${ }^{27}$ A. J. Wurzer, T. Wilhelm, J. Piel, and E. Riedle, Chem. Phys. Lett. 299, 296 (1999).

${ }^{28}$ A. A. Ruth, E.-K. Kim, and A. Hese, Phys. Chem. Chem. Phys. 1, 5121 (1999).

${ }^{29}$ D. Schwarzer, C. Hanisch, P. Kutne, and J. Troe, J. Phys. Chem. A 106, 8019 (2002)

${ }^{30}$ H. Hippler, J. Troe, and H. J. Wendelken, J. Chem. Phys. 78, 5351 (1983).

${ }^{31}$ D. Schwarzer, J. Troe, M. Votsmeier, and M. Zerezke, J. Chem. Phys. 105, 3121 (1996).

${ }^{32}$ D. Schwarzer, J. Troe, and M. Zerezke, J. Chem. Phys. 107, 8380 (1997).

${ }^{33}$ D. Schwarzer, J. Troe, and M. Zerezke, J. Phys. Chem. A 102, 4207 (1998).

${ }^{34}$ T. Wilhelm, J. Piel, and E. Riedle, Opt. Lett. 22, 1494 (1997).

${ }^{35}$ K. Sekiguchi, A. Shimojima, and O. Kajimoto, Chem. Phys. Lett. 356, 84 (2002).

${ }^{36}$ K. Sekiguchi, A. Shimojima, and O. Kajimoto, Chem. Phys. Lett. 370, 303 (2003).

${ }^{37}$ M. Yasunami, S. Miyoshi, N. Kanegae, and K. Takase, Bull. Chem. Soc. Jpn. 66, 892 (1993).

${ }^{38}$ J.-P. Desvergne, N. Bitit, A. Castellan, M. Webb, and H. Bouas-Laurent, J. Chem. Soc., Perkin Trans. 2 1988, 1885 (1988).

${ }^{39}$ D. B. Dess and J. C. Martin, J. Org. Chem. 48, 4155 (1983).

${ }^{40}$ A. G. Anderson, R. G. Anderson, and T. S. Fujita, J. Org. Chem. 27, 4535 (1962).

${ }^{41}$ D. Schwarzer, J. Troe, M. Votsmeier, and M. Zerezke, Ber. Bunsenges. Phys. Chem. 101, 595 (1997).

${ }^{42}$ D. Schwarzer, C. Hanisch, J. Troe, and M. Zerezke, Ultrafast Phenomena XI, edited by T. Elsaesser, J. G. Fujimoto, D. Wiersma, and W. Zinth (Springer, Berlin, 1998), Vol. 63, p. 508.

${ }^{43}$ G. Käb, C. Schröder, and D. Schwarzer (unpublished).

${ }^{44}$ T. Okazaki, N. Hirota, T. Nagata, A. Osuka, and M. Terazima, J. Phys. Chem. A 103, 9591 (1999).

${ }^{45}$ R. E. Peierls, Quantum Theory of Solids (Oxford University Press, London, 1955).

${ }^{46}$ Z. Rieder, L. Lebowitz, and E. Lieb, J. Math. Phys. 8, 1073 (1967).

${ }^{47}$ L. Brouwer, H. Hippler, L. Lindemann, and J. Troe, J. Phys. Chem. 89, 4608 (1985) 